\title{
Tumor of Specialized Prostatic Stroma
}

National Cancer Institute

\section{Source}

National Cancer Institute. Tumor of Specialized Prostatic Stroma. NCI Thesaurus. Code C39904.

A rare neoplasm arising from specialized prostatic stroma. This category includes prostatic stromal proliferations of uncertain malignant potential and prostatic stromal sarcomas. 